\title{
NUMERICAL INVESTIGATION OF TURBULENT FORCED CONVECTIVE FLOWS OVER A PAIR OF CIRCULAR CYLINDERS
}

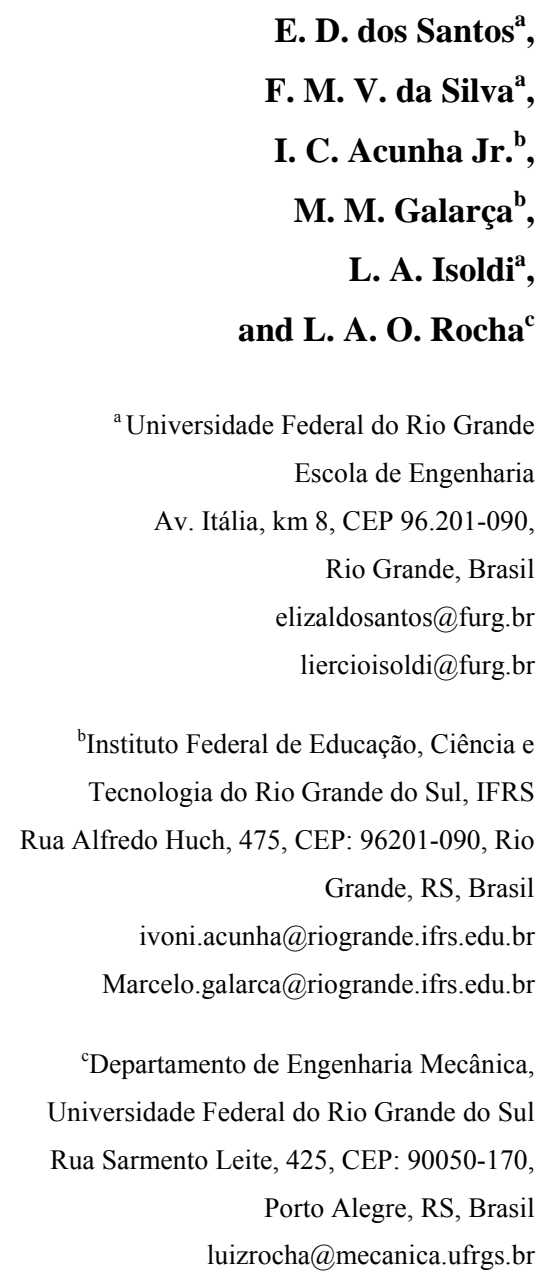

ABSTRACT

The present study presents large eddy simulation (LES) of forced convective heat transfer in transient, two-dimensional, incompressible turbulent flows over a pair of cylinders with two different arrangements: 1) with two circular cylinders in tandem (both cylinders are in line with the streamwise direction of the flow, $\beta=0^{\circ}$ ) and 2) two side-by-side circular cylinders (where both cylinders are placed transversally to the streamwise direction of the flow, $\beta=90^{\circ}$ ). The dynamic Smagorinsky model is employed for the sub-grid treatment. The simulations are based on the finite volume method solution for the conservation equations of mass, momentum and energy. Both simulations are performed with Reynolds and Prandtl numbers of $\operatorname{Re}_{\mathrm{D}}=22000$ and $\mathrm{Pr}=0.71$, respectively. The results showed that the transient fluid dynamic and thermal patterns are strongly affected by the configuration of circular cylinders. The kind of arrangement led to a difference of nearly $20 \%$ for time-averaged Nusselt number $\left(\mathrm{Nu}_{\mathrm{D}}\right)$.

\section{NOMENCLATURE}

A Area, $\mathrm{m}^{2}$

$C_{\mathrm{d}} \quad$ Drag coefficient, $2 F_{\mathrm{d}} /\left(\rho u_{\infty}{ }^{2} A\right)$

$C_{\mathrm{s}}(x, t)$ Smagorinsky constant

$D \quad$ Cylinder diameter, $\mathrm{m}$

$f \quad$ Vortex shedding frequency, $\mathrm{Hz}$

$H \quad$ Height of computational domain, $\mathrm{m}$

$k \quad$ Turbulent kinetic energy, $\mathrm{m}^{2} / \mathrm{s}^{2}$

$k_{\mathrm{c}} \quad$ Minimal cut-off wavenumber, m- 1

$L \quad$ Length of computational domain, $m$

LES Large Eddy Simulation

$\mathrm{Nu}_{\mathrm{D}} \quad$ Nusselt number, $h D / k$

$p \quad$ Distance between cylinders centers, $\mathrm{m}$

$P \quad$ Pressure, $\mathrm{N} / \mathrm{m}^{2}$

Pr Prandtl number, $v / \alpha$

$\operatorname{Pr}_{\mathrm{sgs}}(x, t)$ SGS turbulent Prandtl number

$q^{\prime \prime \prime} \quad$ Heat source per volume, $\mathrm{W} / \mathrm{m}^{3}$

$\operatorname{Re}_{\mathrm{D}} \quad$ Reynolds number, $\rho u_{\infty} D / \mu$

$\bar{S}_{i j} \quad$ Filtered-field deformation tensor, $\mathrm{s}^{-1}$ $\mid \bar{S} \quad$ Strain-rate of the filtered field, $\mathrm{s}^{-1}$

SGS Subgrid-scale

Sth Strouhal number, $f D / u_{\infty}$

$T$ Temperature, $\mathrm{K}$

$t \quad$ Time domain, $\mathrm{s}$

$T_{\mathrm{S}} \quad$ Cylinder surface temperature, $\mathrm{K}$

$u_{\infty} \quad$ Free-stram velocity, $\mathrm{m} / \mathrm{s}$

$u_{\mathrm{w}} \quad$ Wall velocity, $\mathrm{m} / \mathrm{s}$

$v_{i} \quad$ Velocity in $i$-direction, $\mathrm{m} / \mathrm{s}$

$x, y, z \quad$ Cartesian coordinates, $\mathrm{m}$

() Large (filtered) scales

\section{Greek symbols}

$\alpha \quad$ thermal diffusivity, $\mathrm{m}^{2} / \mathrm{s}$

$\alpha_{\mathrm{sgs}} \quad$ thermal eddy diffusivity, $\mathrm{m}^{2} / \mathrm{s}$

$\beta$ angle between the arrangement and the streamwise direction of the flow, degree 
$\delta_{\mathrm{ij}} \quad$ Kronecker delta

$\Delta t \quad$ Time-step, $\mathrm{s}$

$\bar{\Delta} \quad$ subgrid-scale characteristic length, $\mathrm{m}$

$\rho$ density, $\mathrm{kg} / \mathrm{m}^{3}$

$\mu \quad$ dynamical viscosity, $\mathrm{kg} /(\mathrm{m} \cdot \mathrm{s})$

$v \quad$ kinematic viscosity, $\mathrm{m}^{2} / \mathrm{s}$

$v_{\mathrm{sgs}} \quad$ kinematic eddy viscosity, $\mathrm{m}^{2} / \mathrm{s}$

\section{Subscripts}

$\begin{array}{ll}\infty & \text { Stream } \\ \text { w } & \text { Wall } \\ \text { S } & \text { Surface } \\ \text { sgs } & \text { subgrid-scale }\end{array}$

\section{INTRODUCTION}

In spite of extensive experimental and numerical studies almost over a century, flow around a circular cylinder still remains a challenging problem in fluid mechanics and heat transfer, where intensive investigations are continued even today to understand the complex unsteady dynamics of the cylinder wake flow (Çengel and Cimbala, 2006; Rajani et al., 2009). Another motivation for the study of this problem is its large employability for representation of several engineering problems: flows over structures (bridges, towers and buildings), aerodynamic profiles of airplanes, wind energy converters, multi-tubular heat exchangers, condensers, evaporators, steam generators and others (Çengel and Cimbala, 2006; Sahu et al., 2009).

The external flows over circular cylinders or bluff bodies has deserved several attention and many studies have been performed into the numerical and experimental framework with the purpose to evaluate from isothermal laminar flows to forced, natural or mixed convective turbulent flows around one cylinder or bluff body (Rajani et al., 2009; Sahu et al., 2009; Bouris and Bergeles, 1999; Catalano et al., 2003; Franke and Frank, 2002; Perng and Wu, 2007).

Concerning the evaluation of isothermal flows over arrangement of cylinders, Hesam and Navid (2011) evaluated numerically the unsteady, incompressible, two-dimensional laminar and turbulent flows $\left(\operatorname{Re}_{\mathrm{D}}=100,200\right.$ and 10000) around two side-by-side circular cylinders. In this work, turbulence was tackled with the Reynolds-Averaged Navier Stokes (RANS) $k-\varepsilon$ model. Lam and Zoub (2009) evaluated experimentally turbulent isothermal flows around four cylinders in an in-line square configuration with different spacing ratios at Reynolds numbers range of $11000 \leq \mathrm{Re}_{\mathrm{D}} \leq 20000$. Some three-dimensional numerical simulations were also carried out using large eddy simulation (LES). In both studies, the results indicated that several distinct flow patterns exist depending on the spacing ratio (relation between the distance of cylinders and the diameter of them) and Reynolds number of turbulent flow. This behavior also affects the mean values of drag and lift coefficients. Other important studies of turbulent isothermal flows over arrange of bluff bodies and circular cylinders with oscillation have also been reported into literature (Malekzadeh and Sohankar, 2012; Okajima et al., 2007).

For flows with convective heat transfer over arranges of cylinders, Song and Chang (1991) evaluated heat transfer and laminar fluid flow patterns in the mixed convection regimes for the pair of circular cylinders arranged transversally to the vertical air stream. It was observed that the Karman vortex street breaks down behind the heated cylinders in a transient manner for a certain Richardson number due to the buoyancy effect and vortex interaction, in contrast to the sudden breakdown applicable to a single heated cylinder. Into the turbulence framework, it has been observed studies about the influence of transient flow and heat transfer characteristics in wall-mounted matrix of cubes (Meinders and Hanjalić, 1999), stationary ribbed ducts (Sewall and Tafti, 2006) and heated blocks in the channel with transversely oscillating cylinder (Yang and Chen, 2008). However, at the authors knowledge, the influence of the geometry arrange of cylinders over the fluid dynamic and thermal behavior has not been investigated for turbulent flows with heat transfer.

The main purpose of the present work is the numerical investigation of the influence of the cylinders arrangement over the fluid dynamic and thermal behavior of incompressible, transient, turbulent forced convection flows. In this study, it is considered a two-dimensional forced convection turbulent flow over two configurations of the arrangement: 1) two cylinders in-tandem (where both cylinders are placed in line with the streamwise direction of the flow, $\beta=0^{\circ}$ ) and 2) two cylinders side-by-side (where both cylinders are placed transversally to the streamwise direction of the flow, $\beta=90^{\circ}$ ). For both cases, the Reynolds number based on the cylinder diameter and the Prandtl number are kept fixed: $\operatorname{Re}_{\mathrm{D}}=22000$ and $\operatorname{Pr}=0.71$. The simulations of the present study are performed with FLUENT $^{\circledR}$ (Fluent, 2007), which is based on the hexahedral finite volume method for solving the conservation equations of mass, momentum and energy (Patankar, 1980; Versteeg and Malalasekera, 1995). The turbulence is tackled using Large Eddy Simulation (LES) with dynamic Smagorinsky subgrid-scale (DSSGS) (Lesieur et al., 2005; Germano et al., 1991; Lilly, 1992). The latter closure model is selected instead of classical modeling (RANS) due to its higher universality and better application for correct prediction of unsteady phenomena, such as recirculations behind cylinders, shedding of Karman vortices in a wake and KelvinHelmholtz vortices in mixing layer. Examples of these statements are presented in previous studies (Wilcox, 2002; Dos Santos et al., 2011). 


\section{MATHEMATICAL AND NUMERICAL MODELING}

The modeling of transient, incompressible, forced convection turbulent flow is based on the solution of the conservation equations of the problem together with its boundary and initial conditions. In the LES approach, the mass, momentum and energy equations are spatially filtered with a box filter. These equations can be written as (Lesieur et al., 2005):

$$
\begin{gathered}
\frac{\partial \bar{v}_{i}}{\partial x_{i}}=0 \\
\frac{\partial \bar{v}_{i}}{\partial t}+\frac{\partial\left(\bar{v}_{i} \bar{v}_{j}\right)}{\partial x_{j}}=-\frac{1}{\bar{\rho}} \frac{\partial \bar{P}}{\partial x_{j}} \delta_{i j}+\frac{\partial}{\partial x_{j}}\left\{v\left(\frac{\partial \bar{v}_{i}}{\partial x_{j}}+\frac{\partial \bar{v}_{j}}{\partial x_{i}}\right)-\tau_{i j}\right\} \\
\frac{\partial \bar{T}}{\partial t}+\frac{\partial}{\partial x_{j}}\left(\overline{v_{j}} \bar{T}\right)-\frac{\partial}{\partial x_{j}}\left\{\alpha \frac{\partial \bar{T}}{\partial x_{j}}-q_{j}\right\}-\overline{q^{\prime \prime \prime}}=0
\end{gathered}
$$

where () represents the large (filtered) scales, $\rho$ is the fluid density $\left(\mathrm{kg} / \mathrm{m}^{3}\right) ; v$ is the kinematic viscosity $\left(\mathrm{m}^{2} / \mathrm{s}\right) ; \alpha$ is the thermal diffusivity $\left(\mathrm{m}^{2} / \mathrm{s}\right) ; v_{\mathrm{i}}$ is the velocity in $i$-direction, $i=1,2$ and $3(\mathrm{~m} / \mathrm{s}) ; P$ is the pressure $\left(\mathrm{N} / \mathrm{m}^{2}\right) ; T$ is the temperature $(\mathrm{K}) ; \delta_{\mathrm{ij}}$ is the Kronecker delta, $t$ represents the time domain (s); $q^{\prime \prime \prime}$ is the source per volume $\left(\mathrm{W} / \mathrm{m}^{3}\right)$. The terms $\tau_{\mathrm{ij}}$ and $q_{\mathrm{j}}$ that arise in the filtering process of the momentum and energy conservation equations, respectively, need to be modeled and can be written as:

$$
\begin{gathered}
\tau_{i j}=\overline{v_{i} v_{j}}-\bar{v}_{i} \bar{v}_{j} \\
q_{j}=\overline{v_{j} T}-\bar{v}_{j} \bar{T}
\end{gathered}
$$

Concerning the dynamic Smagorinsky subgridscale model (DSSGS), it is based on the hypothesis of Boussinesq's eddy viscosity (Lesieur et al, 2005). For incompressible flows, the turbulent tensor can be written as:

$$
\tau_{i j}=v_{s g s}\left(\frac{\partial \bar{v}_{i}}{\partial x_{j}}+\frac{\partial \bar{v}_{j}}{\partial x_{i}}\right)-\frac{2}{3} k \delta_{i j}
$$

where $v_{\mathrm{sgs}}$ is the kinematic eddy viscosity $\left(\mathrm{m}^{2} / \mathrm{s}\right)$ and $k$ is the turbulent kinetic energy $\left(\mathrm{m}^{2} / \mathrm{s}^{2}\right)$. The turbulent transport of the temperature is obtained by an analogy with the subgrid Reynolds tensor (Lesieur et al., 2005), and is given by:

$$
q_{j}=\alpha_{s g s} \frac{\partial \bar{T}}{\partial x_{j}}
$$

in which $\alpha_{\mathrm{sgs}}$ is the thermal eddy diffusivity $\left(\mathrm{m}^{2} / \mathrm{s}\right)$.

According to the model, the kinematic eddy viscosity and the thermal eddy diffusivity are given by:

$$
\begin{aligned}
& v_{s g s}=C(x, t)^{2} \bar{\Delta}^{2}|\bar{S}| \\
& \alpha_{s g s}=\frac{C(x, t)^{2}}{\operatorname{Pr}_{s g s}(x, t)} \bar{\Delta}^{2}|\bar{S}|
\end{aligned}
$$

where $\bar{\Delta}$ is the subgrid-scale characteristic length (m); $|\bar{S}|$ is the strain-rate of the filtered field $\left(\mathrm{s}^{-1}\right)$ and $\bar{S}_{i j}$ is the filtered-field deformation tensor $\left(\mathrm{s}^{-1}\right)$, which are given by:

$$
\begin{gathered}
\bar{\Delta}=\sqrt[3]{\prod_{i=1}^{3} \Delta x_{i}} \\
|\bar{S}|=\sqrt{2 \bar{S}_{i j} \bar{S}_{i j}} \\
\bar{S}_{i j}=\frac{1}{2}\left(\frac{\partial \bar{v}_{i}}{\partial x_{j}}+\frac{\partial \bar{v}_{j}}{\partial x_{i}}\right)
\end{gathered}
$$

The Smagorinsky constant, $C(x, t)$, and the SGS turbulent Prandtl number, $\operatorname{Pr}_{\mathrm{sgs}}(x, t)$, are dynamically computed based on the approach proposed by Germano et al. (1991) and modified by Lilly (1992). This modeling is based on the use of two spatial filters with different lengths, providing information on the energy transfer between the solved (obtained in the test filtering region) and not solved scales of motion (Lesieur et al., 2005). More details on the SGS model can be found in the works of Lesieur et al. (2005), Germano et al. (1991) and Lilly (1992).

Concerning the numerical approach of turbulent flows, Eqs. (1) to (3) are solved using a CFD package based on hexahedral finite volume method (FVM) (FLUENT $^{\circledR}$ ) (Fluent, 2007). The solver is pressure based and all simulations reported were performed with second-order spatial (bounded central differencing) and implicit temporal discretizations. The bounded central differencing scheme consists of a mixture of two advection schemes: central differencing for regions where the flow is diffusive dominant and upwind of second order for regions where advection is dominant (Gaskell and Lau, 1988; Leonard, 1991; Zhu and Rodi, 1991). The velocitypressure coupling is performed with SIMPLE method. More details about the FVM can be found in the works of Patankar (1980) and Versteeg and Malalasekera (1995). Concerning the solution convergence, the calculations were considered converged when the residuals for the mass, momentum and energy were less than $10^{-5}, 10^{-6}$ and 
$10^{-8}$, respectively. Moreover, an under-relaxation factor of 0.7 was imposed for all conservation equations.

The numerical simulations were performed using a computer with two dual-core Intel processors with $2.67 \mathrm{GHz}$ clock and 8GB of RAM memory. The time processing for the simulations was nearly $2.0 \times$ $10^{6} \mathrm{~s}$.

In order to evaluate the closure model accuracy for the simulation of turbulent external flows, the results obtained here for the Strouhal number (Sth) and the drag coefficient $\left(C_{\mathrm{d}}\right)$ for an isothermal flow around a bluff body of square cross section with $\operatorname{Re}_{\mathrm{D}}$ $=22000$ are compared with the numerical results of Bouris and Bergeles (1999) and experimental of Lyn et al. (1995). The results are presented in Table 1. In comparison with the numerical results of Bouris and Bergeles (1999) the highest difference observed is nearly $3 \%$ for the Strouhal number. All results are also in agreement with the experimental predictions of Lyn et al. (1995).

For cases with convection heat transfer, the method was previously evaluated in the work of Dos Santos et al. (2009). Results showed a close concordance with numerical and experimental results of literature and, for the sake of brevity, will not be repeated here.

Table 1. Comparison of time-averaged parameters for a flow over a square bluff body at $\operatorname{Re}_{\mathrm{D}}=22000$ and $\operatorname{Pr}=0.71$ and those of literature.

\begin{tabular}{ccc}
\hline Reference/Parameter & Sth & Cd \\
\hline Lyn et al. (1995) & $0.132 \pm 0.004$ & $2.05-2.23$ \\
Bouris and Bergeles & 0.134 & 2.18 \\
(1999) & 0.130 & 2.23 \\
Present &
\end{tabular}

\section{PROBLEM DESCRIPTION}

For the problem of this study, it is considered a flow over two cylinders of diameter $D=0.1 \mathrm{~m}$. The distance between the cylinders measured from the center of one cylinder to the center of the other one is $p=1.25 D=0.125 \mathrm{~m}$. Figure 1 depicts the flow domain for the two configurations evaluated: $\beta=0^{\circ}$ (Fig. 1(a)) and $\beta=90^{\circ}$ (Fig. 1(b)). The computational domain has the following dimensions: $L=24.5 \mathrm{D}=$ $2.45 \mathrm{~m}, H=15 D=1.5 \mathrm{~m}, L_{1}=8 D=0.8 \mathrm{~m}$. The fluid flow in the domain is generated by the imposition of constant profile of velocity $u_{\infty}=32 \mathrm{~m} / \mathrm{s}$ (stream velocity) at the inlet surface. The surfaces of the cylinders present the non-slip and the impermeability boundary conditions $\left(u_{\mathrm{w}}=0 \mathrm{~m} / \mathrm{s}\right)$. The superior and inferior surfaces are periodic (in order to avoid the restriction of the free stream flow) and the right lateral surface (named outlet) has an outflow boundary condition. For the thermal field, the heating of the fluid is a result of the temperature difference between the free stream temperature $\left(T_{\infty}=20{ }^{\circ} \mathrm{C}\right)$, which is constant and imposed at the inlet surface, and the temperature of the cylinders $\left(T_{\mathrm{S}}=30^{\circ} \mathrm{C}\right)$.

Concerning the spatial discretization, it is employed a grid with $350 \times 300 \times 1$ in the streamwise $(x)$, normal $(y)$ and spanwise $(z)$ directions, respectively. Moreover, the grid is stretched with a higher refinement near the cylinders surfaces. The minimal cut-off wavenumber for both simulations is $k_{\mathrm{c}}=\pi / \Delta \mathrm{x}=700 \mathrm{~m}^{-1}$. For temporal discretization, it is employed a time-step of $\Delta t=3.0 \times$ $10^{-4} \mathrm{~s}$ or $\Delta t^{*}=\Delta t u_{\infty} / D=3 \times 10^{-2}$. The time-averaged parameters were collected for the flow at the steady state, more precisely from $t=2.3 \mathrm{~s}$ to $t=4.3 \mathrm{~s}$.

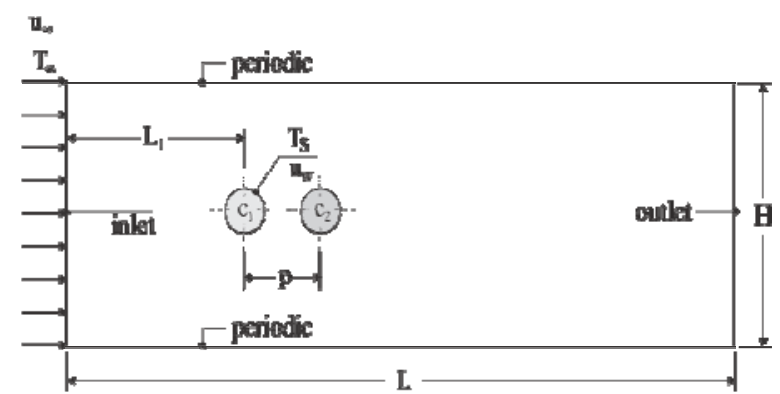

a)

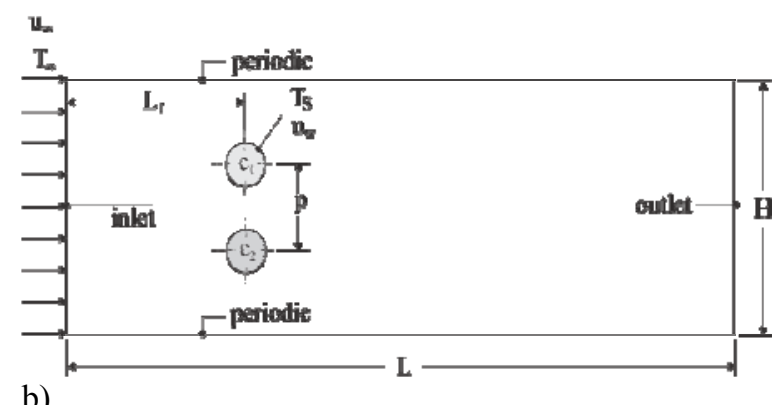

Figure 1. Computational domain of forced convective turbulent flow at $\mathrm{Re}_{\mathrm{D}}=22000$ and $\mathrm{Pr}=0.71$ for two kind of arrangements: (a) $\beta=0^{\circ}$, (b) $\beta=90^{\circ}$.

\section{RESULTS}

Firstly, it is performed a qualitative evaluation of the influence of two arrangements of cylinders over transient behavior of thermal field. Figure 2(a) (d) presents the topologies of the temperature field for the case with $\beta=0^{\circ}$ and for the following dimensionless instants of time: $t^{*}=4.82,9.65,19.30$ and 24.12 (respectively). Figure 3(a) - (d) shows the topologies of temperature field for the case with $\beta=$ $90^{\circ}$ and for the same time steps obtained for the previous case. The temperature ranged from $T_{\infty}=$ $20^{\circ} \mathrm{C}$ (blue color) to $T_{\mathrm{S}}=30^{\circ} \mathrm{C}$ (red color).

In the first case $\left(\beta=0^{\circ}\right)$ it is observed for the initial instant of time (Fig. 2(a)) the formation of two symmetric pair of vortices behind both cylinders $\left(\mathrm{c}_{1}\right.$ and $c_{2}$ ). However, the recirculation region behind cylinder $\mathrm{c}_{1}$ is suppressed due to the presence of the second cylinder $\left(c_{2}\right)$. As a result, the flow in the 
region between the cylinders is also meagered, leading to a poor convective heat transfer in that region. Afterwards, for $t^{*}=9.65$, it is noticed a stretching process and the vortices became asymmetric, similarly to the behavior observed for the flow over only one cylinder. The difference here is caused by the influence of the reattached flow of cylinder $\mathrm{c}_{1}$ over the surface of cylinder $\mathrm{c}_{2}$, which also affects the length of recirculation region behind the cylinder $c_{2}$. In Figure 2(b) it is also observed the stretching of the vortices and the collapse of one of the vortices, in this case the lower one. As time advances, for $t^{*}=19.30$ and $t^{*}=24.12$, it is developed a regular configuration of alternated vortices originating dominant large-scale Kármán vortices. The large eddies are formed at a regular frequency, and they produce disturbances in the flow. It is also seen the increase of the multiplicity of scales due to the transition from laminar to turbulent flow. This behavior has been also reported into literature (Ozgoren et al., 2011). Figure 2(d) presents the vortex street of von Kármán. Moreover, it is observed that for cylinder $c_{2}$ the angle of boundary layer detachment is higher than that noticed for cylinder $\mathrm{c}_{1}$. This fact is a consequence of the increase of momentum caused by the presence of cylinder $c_{1}$. This behavior certainly influences the fluid dynamic and thermal behavior of the flow in comparison with only one cylinder immersed in the flow and is reflected in the drag forces and heat transfer from cylinder to the flow.

For the second case $\left(\beta=90^{\circ}\right)$, it is clearly observed several differences on the thermal field in comparison with the previous case $\left(\beta=0^{\circ}\right)$. For the initial time step $\left(t^{*}=4.82\right)$ it is seen two mixed layers in the upper and lower regions of both cylinders. However, for this case the proximity between both cylinders $\left(c_{1}\right.$ and $\left.c_{2}\right)$ modifies the velocity field that reaches the region between both cylinders. As a consequence, asymmetric recirculations are generated behind each cylinder, differently from what was observed for the previous case $\left(\beta=0^{\circ}\right)$. Afterwards, for the instants of time $t^{*}=9.65$ and $t^{*}=19.30$, the topologies clearly revealed the bistability behavior of the flow over this configuration. It is worthy to mention that, turbulent bistable flows are extremely difficult to be captured by numerical turbulence approaches. The topologies also shown that the detachment of the boundary layer happens before for the case with $\beta=90^{\circ}$ than for the case with $\beta=0^{\circ}$. This fact, probably, conduct to an increase of the drag forces that act over the cylinders. Figure 3 (d) shows two large vortices behind both cylinders, which are stretched generating vortex street of large dimension in the transversal direction, i.e., a higher downstream region can be affected by the vortex street of the cylinders. This recommendation is important for the insertion of new cylinders in the downstream region of domain.

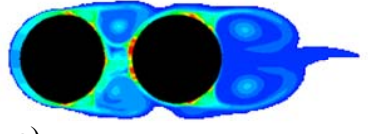

a)

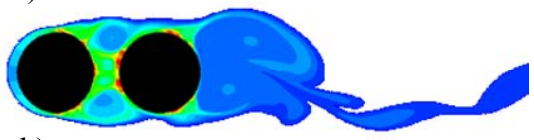

b)
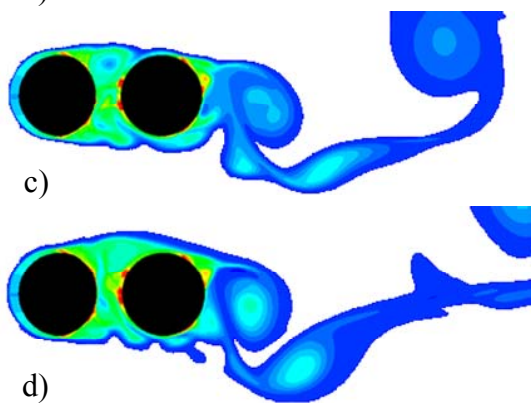

Figure 2. Topologies of the thermal field for $\mathrm{Re}_{\mathrm{D}}=$ $22000, \operatorname{Pr}=0.71$ and $\beta=0^{\circ}$ for the following dimensionless instants of time: (a) $t^{*}=4.82$, (b) $t^{*}=$ 9.65 , (c) $t^{*}=19.30$, (d) $t^{*}=24.12$. a)

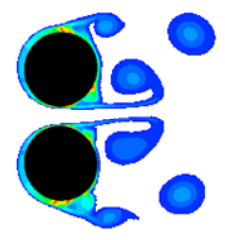

b)

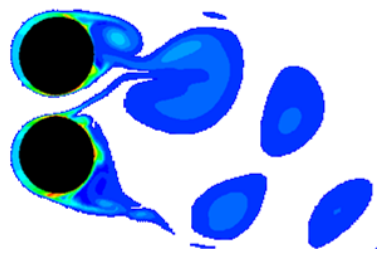

c)
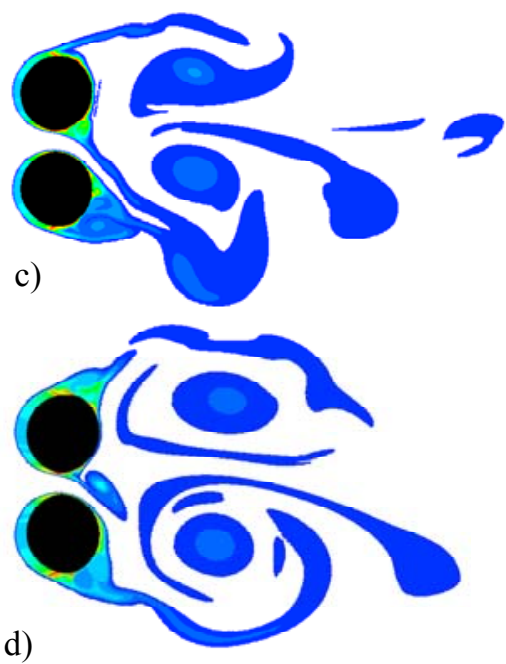

d)

Figure 3. Topologies of the thermal field for $\mathrm{Re}_{\mathrm{D}}=$ 22000, $\operatorname{Pr}=0.71$ and $\beta=90^{\circ}$ for the following dimensionless instants of time: (a) $t^{*}=4.82$, (b) $t^{*}=$ 9.65, (c) $t^{*}=19.30$, (d) $t^{*}=24.12$.

With the purpose to evaluate quantitatively the arrangement influence over the fluid dynamic and thermal fields of the flow, the drag coefficients and Nusselt numbers spatially averaged for the cylinders $\mathrm{c}_{1}$ and $\mathrm{c}_{2}$ are evaluated.

Figure 4(a) shows the spatial averaged coefficient of drag $\left(\mathrm{C}_{\mathrm{d}}\right)$ as a function of 
dimensionless time for the configuration $\beta=0^{\circ}$. As expected, cylinder $c_{1}$ has a higher time-averaged $C_{d}$ than that obtained for cylinder $c_{2}$. The drag coefficient for $c_{1}$ is $C_{d}=0.85$, while for $c_{2}$ is $C_{d}=$ 0.01 . The placement of cylinder $c_{2}$ in a region of low pressure justifies the drastic reduction of its drag coefficient. In spite of the lower magnitude of $\mathrm{C}_{\mathrm{d}}$ for cylinder $c_{2}$, the fluctuation of $\mathrm{C}_{\mathrm{d}}$ for the latter cylinder is higher than that observed for cylinder $c_{1}$. This fact is related with the increase of multiplicity of scales caused by the presence of cylinder $c_{1}$ before cylinder $c_{2}$, i.e., the multiplicity of scales are generated from cylinder $\mathrm{c}_{1}$ to cylinder $\mathrm{c}_{2}$.

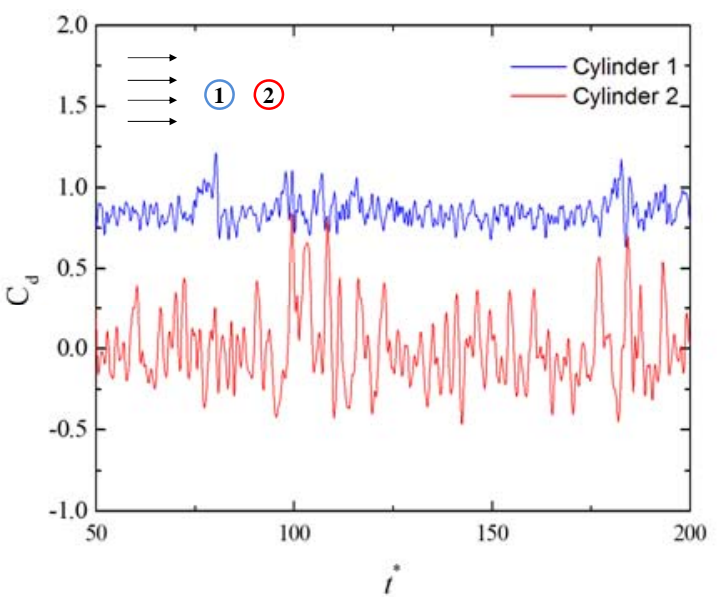

a)

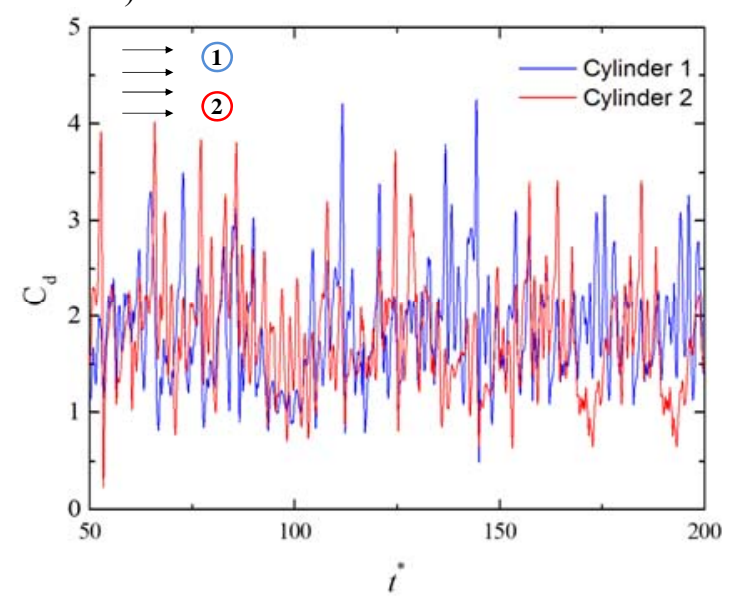

b)

Figure 4. Transient behavior of the drag coefficient

$\left(C_{d}\right)$ in cylinders 1 and 2 for the flow with $\operatorname{Re}_{\mathrm{D}}=$ 22000, $\operatorname{Pr}=0.71$ for two arrangements: (a) $\beta=0^{\circ}$ and (b) $\beta=90^{\circ}$.

Figure 4(b) shows the spatial averaged coefficient of drag $\left(\mathrm{C}_{\mathrm{d}}\right)$ as a function of dimensionless time for the configuration $\beta=90^{\circ}$. For this case, the time-averaged $\mathrm{C}_{\mathrm{d}}$ for both cylinders $\left(\mathrm{c}_{1}\right.$ and $\left.\mathrm{c}_{2}\right)$ has nearly the same magnitude $\left(\mathrm{C}_{\mathrm{d}, \mathrm{c} 1}=1.86\right.$ and $\left.\mathrm{C}_{\mathrm{d}, \mathrm{c} 2}=1.80\right)$. As can be observed, the drag coefficient is higher for $\beta=90^{\circ}$ than for the case with $\beta=0^{\circ}$. For cylinder $c_{1}$, the drag coefficient increases nearly $120 \%$, while for cylinder $\mathrm{c}_{2}$ is observed a step variation, from $C_{d}=0.01$ to $C_{d}=1.80$. Moreover, the fluctuations for both cylinders for the case with $\beta=$ $90^{\circ}$ also increase in comparison with both cylinders for the case with $\beta=0^{\circ}$. For example, the highest fluctuation for cylinder $c_{1}$ and $\beta=90^{\circ}$ is $C_{d}{ }^{\prime}=C_{d, \max }$ $-\mathrm{C}_{\mathrm{d} \text {,ave }}=1.2$. For the case $\beta=0^{\circ}$, the highest fluctuation $\mathrm{C}_{\mathrm{d}}$ ' is $\mathrm{C}_{\mathrm{d}}{ }^{\prime}=0.83$ for cylinder $\mathrm{c}_{2}$. Other important observation was the reflection of the bistable effect of turbulent flow over the computation of drag coefficient. In other words, in some periods of time the $\mathrm{C}_{\mathrm{d}}$ for cylinder $\mathrm{c}_{1}$ increases or decreases while the $C_{d}$ for cylinder $c_{2}$ behaves in opposite direction. This behavior seems more evident for $t^{*} \geq$ 150.

Figure 5(a) shows the spatial averaged Nusselt number as a function of dimensionless time for case $\beta=0^{\circ}$. As observed for $C_{d}$ in Fig. 4(a), the Nusselt number also decreases from cylinder $\mathrm{c}_{1}\left(\mathrm{Nu}_{\mathrm{D}}=\right.$ 120.26) to cylinder $\mathrm{c}_{2}\left(\mathrm{Nu}_{\mathrm{D}}=100.27\right)$. Moreover, the multiplicity of scales generated from cylinder $c_{1}$ to cylinder $c_{2}$ not only affects the fluctuations of $C_{d}$ but also the fluctuations of Nusselt number. The highest fluctuation for cylinder $\mathrm{c}_{2}$ is nearly $50 \%$ higher than that observed for cylinder $\mathrm{c}_{1}$.

Figure 5(b) shows the spatial averaged Nusselt number as a function of dimensionless time for case $\beta=90^{\circ}$. The time-averaged Nusselt number for both cylinders are almost the same $\left(\mathrm{Nu}_{\mathrm{D}}=122.00\right)$. For cylinder $\mathrm{c}_{2}$ it is noticed an increase of $22 \%$ in the time-averaged $\mathrm{Nu}_{\mathrm{D}}$. As observed for coefficient of drag, for the case with $\beta=90^{\circ}$, the bistable effect alternates the magnitude of Nusselt number between the cylinders, which is not observed for the case $\beta=$ $0^{\circ}$. Concerning the fluctuations of Nusselt number, for cylinder $c_{2}$ it is noticed an increase of $24 \%$ in the highest fluctuation from case $\beta=0^{\circ}$ to the case with $\beta$ $=90^{\circ}$.

\section{CONCLUSIONS}

In the present work it was considered a numerical investigation of the influence of the cylinder arrangement over the fluid dynamic and thermal behavior of incompressible, transient, turbulent forced convection flows at $\mathrm{Re}_{\mathrm{D}}=22000$ and $\operatorname{Pr}=0.71$. More precisely, it was considered two configurations of the arrangement: 1) two cylinders in tandem $\left(\beta=0^{\circ}\right)$ and 2$)$ two cylinders side-by-side $\left(\beta=90^{\circ}\right)$. The simulations were performed with FLUENT $^{\circledR}$ (Fluent, 2007), which is based on the hexahedral finite volume method for solving the conservation equations of mass, momentum and energy (Patankar, 1980; Verteeg and Malalasekera, 1995). The turbulence was tackled with LES and dynamic Smagorinsky subgrid-scale (DSSGS) (Lesieur et al., 2005).

The results shown a strong dependence of the transient fluid dynamic and thermal behavior of forced convection turbulent flows for both evaluated 
configurations: $\beta=0^{\circ}$ and $\beta=90^{\circ}$. The transient temperature topologies showed that, the generation of mixing layers, alternated vortices behind the cylinders, the stretched of these vortices and its deliberation in vortex streets of von Kármán were different for both configurations. For the case with $\beta$ $=90^{\circ}$, it was also observed that the flow behaved in a bistable form, which is extremely difficult to be predicted by numerical methods in turbulence.

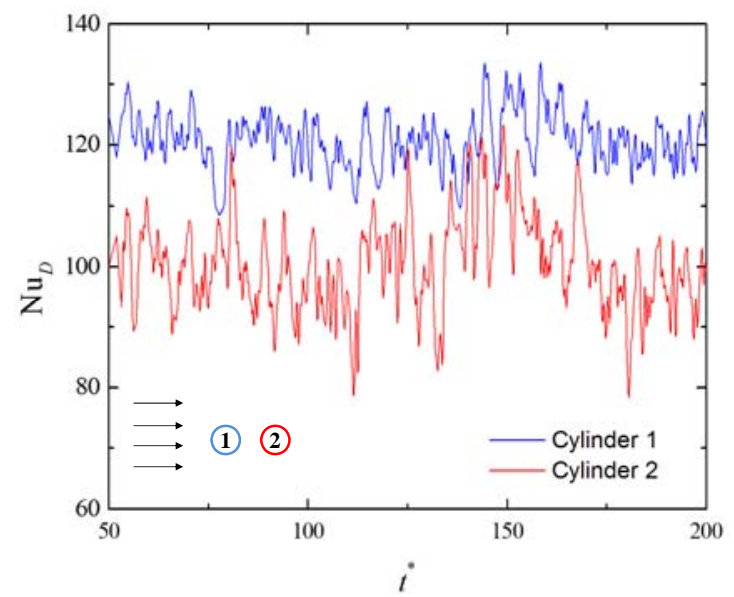

a)

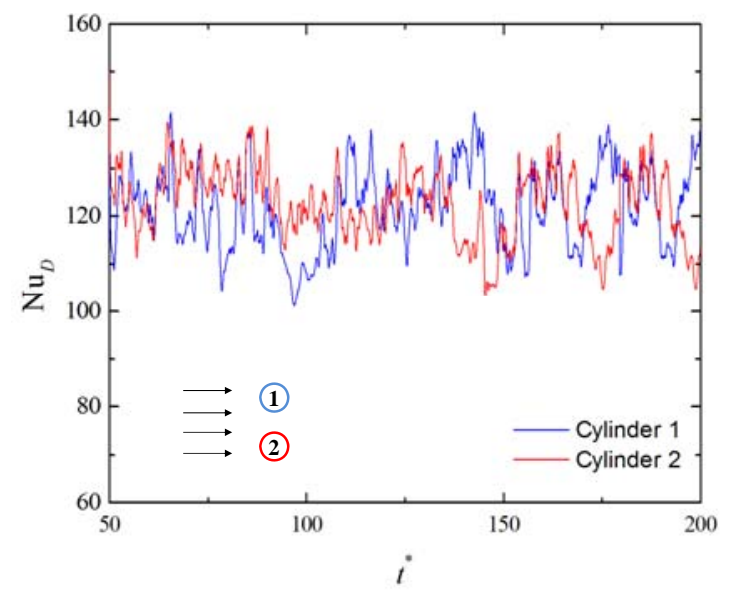

b)

Figure 5. Transient behavior of the Nusselt number $\left(\mathrm{Nu}_{\mathrm{D}}\right)$ in cylinders 1 and 2 for the flow with $\mathrm{Re}_{\mathrm{D}}=$ 22000, $\operatorname{Pr}=0.71$ for two arrangements: (a) $\beta=0^{\circ}$, (b)

$$
\beta=90^{\circ} \text {. }
$$

In order to perform a quantitatively comparison between the two arrangements, the drag coefficient $\left(\mathrm{C}_{\mathrm{d}}\right)$ and Nusselt number $\left(\mathrm{Nu}_{\mathrm{D}}\right)$ for cylinders $\mathrm{c}_{1}$ and $\mathrm{c}_{2}$ were evaluated. For both parameters, it was noticed symmetric values for the case with $\beta=90^{\circ}$ in cylinders $c_{1}$ and $c_{2}$. On the opposite, for the case with $\beta=0^{\circ}$, a step reduction were observed from cylinder $c_{1}$ to cylinder $c_{2}$ (almost $20 \%$ for the Nusselt number). Moreover, the time-averaged and fluctuations of $\mathrm{C}_{\mathrm{d}}$ and $\mathrm{Nu}_{\mathrm{D}}$ also increased from $\beta=0^{\circ}$ to $\beta=90^{\circ}$ in both cylinders. For time-averaged $C_{d}$, it was noticed an increase of nearly $120 \%$ for $\mathrm{c}_{1}$ and a step variation for $c_{2}$. Concerning the Nusselt number, the time-averaged parameter for cylinder $c_{2}$ increased approximately $22 \%$ from $\beta=0^{\circ}$ to $\beta=90^{\circ}$. It is also important to mention that, the bistable behavior of the flow captured for $\beta=90^{\circ}$ was reflected in the transient pattern of drag coefficient and Nusselt number.

\section{ACKNOWLEDGEMENTS}

Professor E. D. dos Santos thanks FAPERGS by financial support (Process: 12/1418-4). Professor L. A. O. Rocha work was sponsored by CNPq, Brasília, Brasil.

\section{REFERENCES}

Bouris, D., and Bergeles, G., 1999, 2D LES of Vortex Shedding from a Square Cylinder, Journal of Wind Engineering and Industrial Aerodynamics, Vol. 80, pp. 31-46.

Catalano, P., Wang, M., Iaccarino, G., and Moin, P., 2003, Numerical Simulation of the Flow Around a Circular Cylinder at High Reynolds numbers, International Journal of Heat and Fluid Flow, Vol. 24, pp. 463-469.

Çengel, Y. A., and Cimbala, J. M., 2006, Fluid Mechanics: Fundamentals and Application. New York: McGraw-Hill.

Dos Santos, E. D., Dall'Agnol, A., Petry, A. P., and Rocha, L. A. O., 2009, Heat Transfer Optimization of Cross-Flow over Assemblies of Bluff Bodies Employing Constructal Principle, in: Proc. 20th International Congress of Mechanical Engineering, Gramado, Brasil, pp. 1-8.

Dos Santos, E. D., Piccoli, G. L., França, F. H. R., and Petry, A. P., 2011, Analysis of Mixed Convection in Transient Laminar and Turbulent Flows in Driven Cavities, International Journal of Heat and Mass Transfer, Vol. 54, pp. 4585-4595.

Fluent (version 6.3.16), 2007, ANSYS, Inc.

Franke, J., and Frank, W., 2002, Large Eddy Simulation of the Flow Past a Circular Cylinder at $\mathrm{Re}_{\mathrm{D}}=3900$, Journal of Wind Engineering and Industrial Aerodynamics, Vol. 90, pp. 1191-1206.

Gaskell, P. H., and Lau, A. K. C., 1988, Curvative-Compensated Convective Transport: SMART, a New Boundedness-Preserving Transport Algorithm, International Journal for Numerical Methods in Fluids, Vol. 8 (6), pp. 617-641.

Germano, M., Piomelli, U., Moin, P., and Cabot, W. H., 1991, A Dynamic Sub-Grid-Scale Eddy Viscosity Model, Physics of Fluids A, Vol. 3, pp. 1760-1765.

Hesam, S.-M., and Navid, N., 2011, Numerical Simulation of Flow Over Two Side-by-Side Circular Cylinders, Journal of Hydrodynamics, Vol. 23 (6), pp. 792-805.

Lam, K., and Zoub, L., 2009, Experimental Study and Large Eddy Simulation for the Turbulent 
Flow Around Four Cylinders in an In-Line Square Configuration, International Journal of Heat and Fluid Flow, Vol. 30, pp. 276-285.

Leonard, B. P., 1991, The Ultimate Conservative Difference Scheme Applied to Unsteady One-dimensional Advection, Computational Methods Applied Mechanics Engineering, Vol. 88, pp. 17-74.

Lesieur, M., Métais, O., and Comte, P., 2005, Large-eddy Simulations of Turbulence, Cambridge, New York.

Lilly, D. K., 1992, A Proposal Modification of the Germano Subgrid-Scale Closure Method, Physics of Fluids A, Vol. 4, pp. 633-635.

Lyn, D., Elinav, S., Rodi, W., and Park, J., 1995, A Laser Doppler Velocimetry Study of Ensemble-Averaged Characteristics of the Turbulent Near Wake of a Square Cylinder, Journal of Fluid Mechanics, Vol. 304, pp. 285-319.

Malekzadeh, S., and Sohankar, A., 2012, Reduction of Fluid Forces and Heat Transfer on a Square Cylinder in a Laminar Flow Regime Using a Control Plate, International Journal of Heat and Fluid Flow, Vol. 34, pp. 15-27.

Meinders, E. R., and Hanjalić, K., 1999, Vortex Structure and Heat Transfer in Turbulent Flow Over a Wall-Mounted Matrix of Cubes, International Journal of Heat and Fluid Flow, Vol. 20, pp. 255-267.

Okajima, A., Yasui, S., Kiwata, T., and Kimura, S., 2007, Flow-induced Streamwise Oscillation of Two Circular Cylinders in Tandem Arrangement, International Journal of Heat and Fluid Flow, Vol. 28 , pp. 552-560.

Ozgoren, M., Pinar, E., Sahin, B., and Akilli, H., 2011, Comparison of Flow Structures in the Downstream Region of a Cylinder and Sphere, International Journal of Heat and Fluid Flow, Vol. 32, pp. 1138-1146.

Patankar, S. V., 1980. Numerical Heat Transfer and Fluid Flow, McGraw Hill, New York.

Perng, S-W., and Wu, H-W., 2007, BuoyancyAided/Opposed Convection Heat Transfer for Unsteady Turbulent Across a Square Cylinder in a Vertical Channel, International Journal of Heat and Mass Transfer, Vol. 50, pp. 3701-3717.

Rajani, B. N., Kandasamy, A., and Majumdar, S., 2009, Numerical Simulation of Laminar Flow Past a Circular Cylinder, Applied Mathematical Modelling, Vol. 33, pp. 1228-1247, 2009.

Sahu, A. K., Chhabra, R. P., and Eswaran, V., 2009, Effects of Reynolds and Prandtl Numbers on Heat Transfer from a Square Cylinder in the Unsteady Flow Regime, International Journal of Heat and Mass Transfer, Vol. 52, pp. 839-850.

Sewall, E. A., and Tafti, D. K., 2006, Experimental Validation of Large Eddy Simulations of Flow and Heat Transfer in a Stationary Ribbed Duct, International Journal of Heat and Fluid Flow, Vol. 27, pp. 243-258.
Song, C-J., Chang, K-S., 1991, Heat Transfer and Interactive Buoyant Vortex Sheeding by a Pair of Circular Cylinders in Transverse Arrangement, International Journal of Heat and Mass Transfer, Vol. 34 (6), pp. 1347-1354.

Versteeg, H. K., and Malalasekera, W., 1995, An Introduction to Computational Fluid DynamicsThe Finite Volume Method, Longman, England.

Wilcox, D.C., 2002, Turbulence Modeling for $C F D, 2^{\text {nd }}$ ed., DCW Industries, La Canada.

Yang, Y-T., and Chen, C-H., 2008, Numerical Simulation of Turbulent Fluid Flow and Heat Transfer Characteristics of Heated Blocks in the Channel with an Oscillating Cylinder, International Journal of Heat and Mass Transfer, Vol. 51, pp. 1603-1612.

Zhu, J., and Rodi, W., 1991, A Low Dispersion and Bounded Convection Scheme, Computational Methods Applied Mechanics Engineering, Vol. 92, pp. 225-232.

Received: September 30, 2012

Revised: October 30, 2012

Accepted: November 30, 2012 